\title{
Mitigating Large Background in Jet Substructure Observables
}

\author{
Daekyoung Kang ${ }^{1, \star}$ Yang-Ting Chien ${ }^{2, \star \star}$ Kyle Lee ${ }^{3,4, \star \star \star}$ Yiannis Makris ${ }^{5, \star \star \star \star}$ \\ ${ }^{1}$ Key Laboratory of Nuclear Physics and Ion-beam Application (MOE) and Institute of Modern Physics, Fudan \\ University, Shanghai, China 200433 \\ ${ }^{2}$ Center for Theoretical Physics, Massachusetts Institute of Technology, Cambridge, MA 02139, USA \\ ${ }^{3}$ C.N. Yang Institute for Theoretical Physics, Stony Brook University, Stony Brook, NY 11794, USA \\ ${ }^{4}$ Department of Physics and Astronomy, Stony Brook University, Stony Brook, NY 11794, USA \\ ${ }^{5}$ Theoretical Division T-2, Los Alamos National Laboratory, Los Alamos, NM, 87545, USA
}

\begin{abstract}
We discuss a new approach for jet physics analysis by using subtraction between cumulants of jet substructure observables. The subtracted cumulants are insensitive to soft-particle background uncorrelated with the hard process and allow comparisons between theoretical results and experimental measurements without the complication of soft background like underlying and pile-up events. We find our method using jet mass cumulants efficiently eliminates the background in Monte Carlo simulations and ATLAS jet mass measurements and they show a good agreement with our analytic calculations performed using soft-collinear effective theory.
\end{abstract}

\section{Introduction}

Jets produced everywhere in high-energy process are one of essential objects of study at high energy colliders. However, theoretical predictions are many times limited by soft radiations such as multiparton interactions (MPI) in hadron collisions and pile-up (PU) events in high-luminosity (HL) collisions. The predictions cannot be compared with experiments without plugging in model for the soft radiations. Or, one has to adopt techniques [1-10] developed to remove these contributions. Ideal goal of these techniques would be to correct jets back to their pure signal jet but it is impossible due to the intrinsic ambiguity in distinguishing signal from the background. In Ref. [11], we proposed an alternative approach using subtraction between cumulants which cancel the contributions from soft uncorrelated emissions (SUEs) and applied for the transverse energy observable of DrellYan processes. Then, we further applied this method to jet substructure observables in [12]. For the observables to which SUEs additively contribute, their contributions are cancelled in the subtraction between two cumulants. As a concrete example for this, we used the classic jet observable, jet mass $m_{J}$, and showed theoretical predictions for subtracted jet mass cumulant $\Delta$ agree with Monte Carlo simulations and with ATLAS measurements.

\footnotetext{
^e-mail: dkang@fudan.edu.cn

$\star \star$ e-mail: ytchien@mit.edu

$\star \star \star$ e-mail: kunsu.lee@stonybrook.edu

$\star \star \star \star$ e-mail: yiannis@lanl.gov
} 
In this proceeding, we review and summarize of our result in [12]. In following sections we first define the subtracted cumulant $\Delta$, then show that in Monte Carlo simulations the subtraction is robust against various SUE effect. We also show that our theoretical predictions are in a good agreement with ATLAS measurements in subtracted cumulant space.

\section{Definition of the observable}

Let us consider a jet substructure observable, $e$, which can be expressed as an addition of individual particle contribution. Then, SUEs contribution can be separated from signal contribution as

$$
e=e_{\mathrm{S}}+e_{\mathrm{B}}
$$

where $e_{\mathrm{B}}$ is background contribution by SUEs and it is statistically independent of $e_{\mathrm{S}}$. In other words, their probability densities are uncorrelated each other by the definition of SUEs. The cumulants $\kappa_{n}(e)$ is defined by its generating function: $\sum_{\mathrm{n}=1}^{\infty} \kappa_{n} \frac{t^{n}}{n !}=\log \langle\exp (t e)\rangle$. The cumulants inherit the additivity of observable and the background contribution remains separated.

$$
\kappa_{n}(e)=\kappa_{n}\left(e_{\mathrm{S}}\right)+\kappa_{n}\left(e_{\mathrm{B}}\right)
$$

We use this property to cancel uncorrelated contributions in the subtraction between cumulants.

Let us define an additive observable $\hat{\tau}$

$$
\hat{\tau}=2 \cosh (\eta) \sum_{i \in \mathrm{jet}} p_{i}^{+}=\frac{m_{J}^{2}}{p_{T}}\left[1+O\left(\frac{m_{J}^{2}}{p_{T}^{2}}\right)\right],
$$

where $\eta$ is pseudorapidity of jet and $p_{T}$ is the jet transverse momentum. This can be written as an addition of signal and background as in Eq. (1) and is equivalent to the jet invariant mass $m_{J}^{2} / p_{T}$ up to relative corrections of $\left(m_{J} / p_{T}\right)^{2}$.

For the dimensionless version $\tau=\hat{\tau} / p_{T}$, the first cumulant $\langle\tau\rangle$ is given by

$$
\langle\tau\rangle=\left(\frac{d \sigma}{d p_{T} d \eta}\right)^{-1} \int d \tau \tau \frac{d \sigma}{d \tau d p_{T} d \eta}=\left\langle\tau_{\text {corr }}\right\rangle+\frac{\Omega_{f}}{p_{T}},
$$

where $\left\langle\tau_{\text {corr }}\right\rangle$ is the signal contribution correlated with hard scale, while $\Omega_{f}$ is the background and uncorrelated with hard scale.

For comparison with experimental results, we consider a binned cross section $\sigma^{[i, j]}$ where the first index represents the $i$-th bin in $\tau$ and the second represents $j$-th bin in jet $p_{T}$. The cumulant at $j$-th $p_{T}$ bin is denoted by $\langle\tau\rangle^{[j]}$. Now let us define the subtracted cumulant of $\tau$

$$
\Delta_{\tau}^{j k}=\langle\tau\rangle^{[j]}-\langle\tau\rangle^{[k]} \frac{\left\langle p_{T}^{-1}\right\rangle^{[j]}}{\left\langle p_{T}^{-1}\right\rangle^{[k]}}=\left\langle\tau_{\text {corr }}\right\rangle^{[j]}-\left\langle\tau_{\text {corr }}\right\rangle^{[k]} \frac{\left\langle p_{T}^{-1}\right\rangle^{[j]}}{\left\langle p_{T}^{-1}\right\rangle^{[k]}} .
$$

Here the background contribution $\Omega_{f}$ is cancelled in the subtraction and the subtraction is rewritten in terms of pure signal contribution $\tau_{\text {corr }}$.

\section{Robustness of subtracted cumulant}

\subsection{Pythia simulations}

Here, we verify that the subtracted cumulants are robust again to the background such as MPI and PU by using PyтніA simulations in $p p$ collisions. We also compare that the subtracted cumulants obtained 


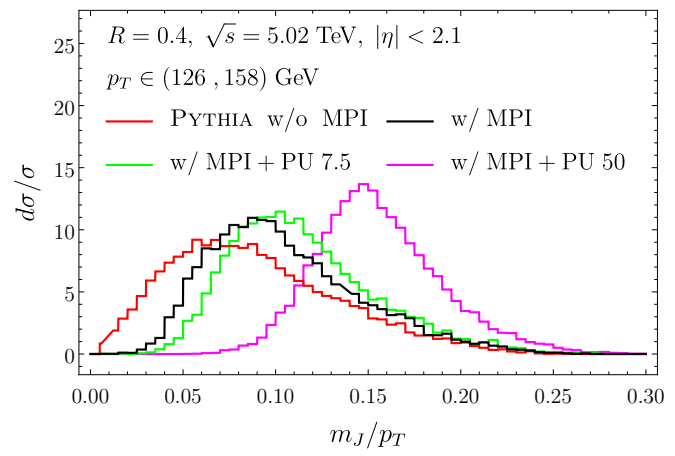

Figure 1. Jet mass distributions using PyтнIA at parton level (red), hadron level with MPI (black), PU with $\left\langle N_{\mathrm{PU}}\right\rangle=7.5$ (green) and 50 (magenta).

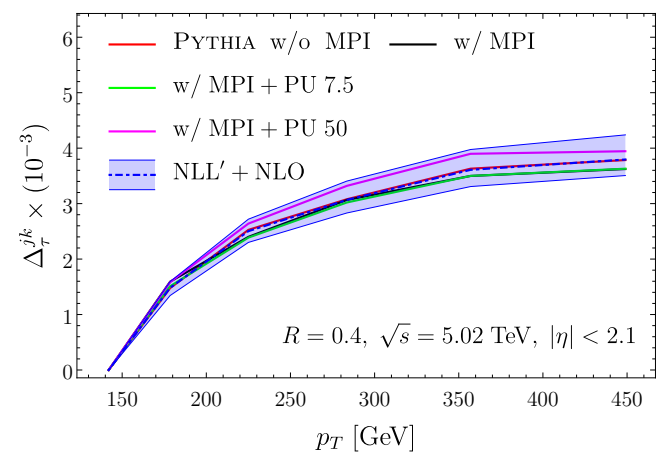

Figure 2. Subtracted cumulants $\Delta_{\tau}^{j k}$ for jet mass for $p_{T}$ between 126 and $500 \mathrm{GeV}$. perturbative calculation (blue band) and PYтHIA simulations.

by the simulation agree with theoretical predictions at next-to-leading logarithmic and next-to-leading order accuracy (NLL'+NLO) using SCET.

FIG. 1 shows the jet mass distributions with and without MPI and PU effects. We find significant changes in the distribution by MPI and PU. On the other hand, subtracted cumulants in FIG. 2, remains almost same except for large PU (PU 50 curve). For the large PU, $p_{T}$ is also shifted by the SUEs and to improved the results, a modified definition of $p_{T}$ robust against SUEs can be adopted [12]. The simulation results also agree with theoretical prediction within perturbative uncertainty (blue band). This clearly verifies that our subtracted cumulants greatly mitigate MPI and PU contributions.

\subsection{Comparison with experimental data}

Here, we compare the theoretical predictions and simulations to LHC data measured by ATLAS collaboration at $7 \mathrm{TeV}$ [13] and 5.02 TeV [14]. FIG. 3 shows the results for the predictions at NLL'+NLO accuracy and PYтнIA simulations with and without MPI. The data points are for ATLAS results. Note that in $7 \mathrm{TeV}$ data [13], the jet mass $s=m_{J}^{2}$ is given rather than $\tau=m_{J}^{2} / p_{T}^{2}$, we rescale the definition of $\Delta$ and denote it to $\Delta_{s}$

$$
\Delta_{s}^{j k}=\langle s\rangle^{[j]}-\langle s\rangle^{[k]} \frac{\left\langle p_{T}\right\rangle^{[j]}}{\left\langle p_{T}\right\rangle^{[k]}} .
$$



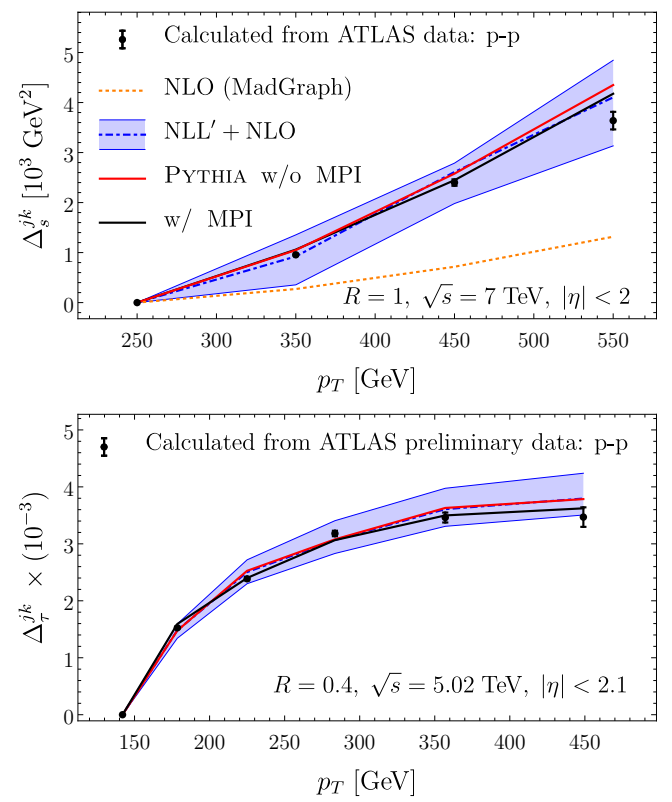

Figure 3. subtracted cumulant results: ATLAS results (data points) computed using data in $[13,14], \mathrm{NLL}^{\prime}+\mathrm{NLO}^{\prime}$ calculation (blue band), PYTHIA simulations with and without MPI (black and red curves). The top and bottom panels are for $\sqrt{s}=7 \mathrm{TeV}$ and $\sqrt{s}=5.02 \mathrm{TeV}$, respectively.

We find that both of $5 \mathrm{TeV}$ and $7 \mathrm{TeV}$ data points are in good agreement with our predictions as well as simulations.

\section{Summary}

In this proceeding, we review and summarize our results in $[11,12]$. We introduce a new method for jet substructure observables called a subtracted cumulant. This quantity is insensitive to soft emissions uncorrelated with hard process hence, robust against multiparton interactions and pile-up effects. The is independent way of studying jet substructure from conventional techniques which either correct jets by removing soft constituents or use a model for soft emissions. In order to verify this method, we computed the subtracted cumulant of jet mass at $\mathrm{NLL}^{\prime}+\mathrm{NLO}$ accuracy using SCET and compared it to those obtained from Pyтнia simulations. While in theoretical prediction the soft uncorrelated emissions is absent, in simulations those emissions are turned on and they induce significant changes in the jet mass distributions. However subtracted cumulants of theoretical predictions and simulations still remain in a good agreement. This verifies the subtraction method mitigates large background. Finally, we test it with ATLAS measurements and find a similar agreement with theory predictions.

\section{References}

[1] M. Cacciari, G.P. Salam, Phys. Lett. B659, 119 (2008), 0707. 1378

[2] I. Feige, M.D. Schwartz, I.W. Stewart, J. Thaler, Phys. Rev. Lett. 109, 092001 (2012), 1204.3898

[3] A.J. Larkoski, J. Thaler, Phys. Rev. D90, 034010 (2014), 1406.7011 
[4] D. Krohn, M.D. Schwartz, M. Low, L.T. Wang, Phys. Rev. D90, 065020 (2014), 1309. 4777

[5] M. Cacciari, G.P. Salam, G. Soyez, Eur. Phys. J. C75, 59 (2015), 1407.0408

[6] D. Bertolini, P. Harris, M. Low, N. Tran, JHEP 10, 059 (2014), 1407.6013

[7] P. Berta, M. Spousta, D.W. Miller, R. Leitner, JHEP 06, 092 (2014), 1403 . 3108

[8] A.J. Larkoski, S. Marzani, G. Soyez, J. Thaler, JHEP 05, 146 (2014), 1402.2657

[9] P.T. Komiske, E.M. Metodiev, B. Nachman, M.D. Schwartz, JHEP 12, 051 (2017), 1707.08600

[10] G. Soyez, Phys. Rept. 803, 1 (2019), 1801.09721

[11] D. Kang, Y. Makris, T. Mehen, JHEP 09, 055 (2018), 1803.04413

[12] Y.T. Chien, D. Kang, K. Lee, Y. Makris, Phys. Rev. D100, 074030 (2019), 1812.06977

[13] G. Aad et al. (ATLAS), JHEP 05, 128 (2012), 1203.4606

[14] The ATLAS collaboration (ATLAS), ATLAS-CONF-2018-014 (2018) 